\title{
Ecology of dark matter haloes -II. Effects of interactions on the alignment of halo pairs
}

\author{
Benjamin L'Huillier, ${ }^{1,2}$ Changbom Park ${ }^{1}$ and Juhan Kim ${ }^{3 \star}$ \\ 1 School of Physics, Korea Institute for Advanced Study, 85 Hoegi-ro, Dongdaemun-gu, Seoul 130-722, Korea \\ 2 Korea Astronomy and Space Science Institute, 776 Daedeok daero, Yuseong-gu, Daejeon, Korea \\ ${ }^{3}$ Center for Advanced Computation, Korea Institute for Advanced Study, 85 Hoegi-ro, Dongdaemun-gu, Seoul 130-722, Korea
}

Accepted 2017 January 16. Received 2017 January 13; in original form 2016 March 20

\begin{abstract}
We use the Horizon Run 4 cosmological $N$-body simulation to study the effects of distant and close interactions on the alignments of the shapes, spins, and orbits of targets haloes with their neighbours, and their dependence on the local density environment and neighbour separation. Interacting targets have a significantly lower spin and higher sphericity and oblateness than all targets. Interacting pairs initially have anti-parallel spins, but the spins develop parallel alignment as time goes on. Neighbours tend to evolve in the plane of rotation of the target, and in the direction of the major axis of prolate haloes. Moreover, interactions are preferentially radial, while pairs with non-radial orbits are preferentially prograde. The alignment signals are stronger at high-mass and for close separations, and independent on the large-scale density. Positive alignment signals are found at redshifts up to 4, and increase with decreasing redshifts. Moreover, the orbits tend to become prograde at low redshift, while no alignment is found at high redshift $(z=4)$.
\end{abstract}

Key words: Methods: numerical - Galaxies: haloes, interactions - Cosmology: Largescale structure of the Universe, Theory, Dark matter

\section{INTRODUCTION}

Galaxy surveys and cosmological simulations have revealed that the Universe is structured on large-scale as a cosmic web made of two-dimensional walls and one-dimensional filaments encompassing voids, with clusters in the knots (de Lapparent et al. 1986). This cosmic web is the natural outcome of the gravitational collapse of the primordial density fluctuations (Bond et al. 1996). Galaxies evolve in this cosmic web, accreting material along the filaments. The anisotropic nature of the cosmic web is thus expected to affect the development of galaxy properties, such as the acquisition of their angular momentum or their orientation. Understanding the way galaxies acquire their spin and shapes can thus shed light on galaxy formation. Intrinsic galaxy alignments are also a source of systematics for upcoming lensing surveys (see Joachimi et al. 2015 for a recent review).

The tidal torque theory (TTT, Hoyle 1951; Doroshkevich 1970; White 1984; Lee \& Pen 2000) states that protogalaxies acquire their angular momentum by the gravitational torque due to the misalignment of their inertia tensor and the tidal tensor due to the large-scale structures. It then

* E-mail:benjamin@kasi.re.kr (BL), cbp@kias.re.kr (CBP), kjhan@kias.re.kr (JHK) predicts that the spin should be aligned with the intermediate axis (orthogonal to the filaments and in the plane of the walls) of the tidal tensor. However, this theory is only valid in the linear to mildly non-linear regime, and cannot predict accurately the eventual spin of the collapsed halo (Porciani et al. 2002).

$N$-body simulations have shown that haloes in walls have their spins in the plane of the wall, confirming the TTT (Hahn et al. 2007; Aragón-Calvo et al. 2007). However, they also showed that the situation in filaments is massdependent. Low-mass haloes have their spins aligned with the direction of the filaments, while massive ones are orthogonal (Hahn et al. 2007; Aragón-Calvo et al. 2007; Codis et al. 2012; Hoffman et al. 2012; Libeskind et al. 2013b; Trowland et al. 2013; Aragon-Calvo \& Yang 2014). In a detailed study of the halo-filament alignments, Codis et al. (2012) argued that low-mass haloes build their mass from smooth accretion along the filaments, and therefore acquire a spin parallel to it, while the more massive ones undergo mergers that tend to build a spin orthogonal to the filament. Laigle et al. (2015) showed that the origin of the spin is due to the vortices in the filaments.

Simulations showed that the major axis of the halo has a stronger alignment signal with the LSS (direction of filament, or in the plane of the wall) (Aragón-Calvo et al. 2007; 
Zhang et al. 2009; Libeskind et al. 2013a; Forero-Romero et al. 2014), the alignment signal being stronger for more massive haloes. Using the tidal web, Forero-Romero et al. (2014) found a strong alignment of the major axis of the haloes with the direction of the filaments and the normal of the walls as defined by the tidal field. Interestingly, when defining the LSS using a mass-weighted velocity shear tensor, they found an anti-alignment between the major axis of the halo and the direction of the filament or normal to the wall.

However, galaxies are not only sensitive to the gravitational component, but also governed by baryonic processes. Thanks to recent progress in modelling the baryonic processes involved in galaxy formation as well as an increase of computing power leading to better resolution, galaxy alignments have also been investigated in hydrodynamical simulations (Velliscig et al. 2015a,b; Chen et al. 2015; Chisari et al. 2015; Welker et al. 2015; Shao et al. 2016). Using the Horizon-AGN simulation (Dubois et al. 2014), Chisari et al. (2015) found two alignment signals, corresponding to elliptical and spiral galaxies.

Observationally, the picture is not so clear (Lee \& Pen 2002; Lee et al. 2005; Yang et al. 2006; Aryal et al. 2007; Lee \& Erdogdu 2007; Wang et al. 2009; Lee 2011; Zhang et al. 2013, 2015). Lee (2011) found a stronger correlation of the spin of spiral galaxies than predicted by the TTT, implying a misalignment between the galaxy and the dark halo. Yang et al. (2006) using the SDSS DR2 found that the positions of satellites are aligned with the major axis of the group, with a stronger alignment for red centrals and satellites, for higher halo mass, and at smaller radii. These results were extended by Wang et al. (2009) who found that neighbouring groups tend to be aligned with the major axis of the target groups. Zhang et al. (2013) using the SDSS DR7 found an alignment signal between the major axis of central galaxies and the direction of filaments and the plane of walls, while no signal was found for satellites. The alignment of red centrals is of the same order as that of the halo, while blue centrals show a weaker alignment. (Tempel \& Tamm 2015) studied the alignment of galaxy pairs with the filaments, and found that loose pairs $(d>0.3 \mathrm{Mpc})$ are more aligned with the direction of the filaments than close pairs.

However, reliably defining the LSS in observation is a difficult task. Alternatively, one may look at alignments in halo pairs. In our previous paper (L'Huillier et al. 2015, hereafter Paper I), we studied the rate at which targets undergo an interaction with a neighbour at least 0.4 times as massive. In this study, we used the same data and method to investigate the alignment of spin and shapes of interacting pairs.

Section 2 briefly presents the simulation (Kim et al. 2015) and the catalogue (Paper I), § 3 shows our main results in terms of alignments of spins, shapes, and orbits of interacting pairs, and the conclusions are drawn in $\S 4$.

\section{SIMULATION AND METHOD}

\subsection{The Horizon Run 4 simulation}

We used the Horizon Run 4 simulation (Kim et al. 2015), a massive $N$-body simulation with $N=6300^{3}$ particles in a
$L=3.15 h^{-1} \mathrm{Gpc}$ box in a flat WMAP5 $\Lambda$-cold dark matter cosmology, starting at $z=100$ with second order Lagrangian perturbation theory, ensuring a 1\%-level accurate power spectrum and halo mass function (L'Huillier et al. 2014). Haloes were detected using the Ordinary Parallel Friends-of-Friends algorithm (OPFoF, Kim et al. 2015) with a linking length of 0.2 times the mean particle separation, and the gravitationally-bound subhaloes, which are assumed to host galaxies, with the PSB subhalo finder (Kim \& Park 2006). The PSB subhalo finding method is similar to SUBFIND (Springel et al. 2001) using peak finding and density gradient to allocate members to the subhalo candidates. Additionally, PSB uses tidal boundaries to demarcate each subhalo region. For more details, we refer the readers to Kim \& Park (2006). Hereafter, we will refer to PSB subhaloes as haloes.

\subsection{Catalogue and definitions}

The catalogue was described in Paper I. Our target (T) catalogue consists of all haloes more massive than $M=$ $5 \times 10^{11} h^{-1} \mathrm{M}_{\odot}$, while the neighbour catalogue $(\mathrm{N})$ consists of those more massive than $2 \times 10^{11} h^{-1} \mathrm{M}_{\odot}$, corresponding respectively to 56 and 23 particles. A target of mass $M_{\mathrm{T}}$ is defined to be interacting if it is located within the virial radius of its nearest neighbour of mass $M_{\mathrm{N}}>\xi_{0} M_{\mathrm{T}}$, with $\xi_{0}=0.4$. Therefore, the maximum separation allowed to an "interacting" halo pair depends on the virial radius of the neighbour halo. This choice was made in accordance with the idea that a halo should be regarded interacting when it is under the significant influence of its nearest neighbour. It is also based on the observational finding that the effect of the morphological type of the neighbour starts affecting that of the target only when the pair separation between the members is shorter than $R_{\mathrm{vir}, \mathrm{N}}$ (see Fig. 6 of Park \& Choi 2009). Note that in this definition, we are counting interactions per target halo rather than per pair. Therefore, some interactions are counted twice, e.g., if the target is also the neighbour's nearest neighbour, but it is not necessarily the case.

To quantify the environment, in addition to the target mass, we use the large-scale density smoothed over the 20 nearest neighbours

$$
\rho_{20}=\sum_{1}^{20} M_{i} W\left(r_{i} / h\right),
$$

where $M_{i}$ is the mass of the $i$ th neighbour, $r_{i}$ the distance between the target and the $i$ th neighbour, $h$ the smoothing scale chosen to enclose the 20 closest neighbours, and $W$ the cubic B-spline smoothing kernel used in smooth particle hydrodynamics simulations (Monaghan \& Lattanzio 1985). The density is then normalized to a dimensionless parameter $\delta$ by

$$
1+\delta=\frac{\rho_{20}}{\bar{\rho}}
$$

where

$$
\bar{\rho}=\frac{1}{V} \sum_{i \in \mathrm{N}} M_{i}
$$

is the mean density of the neighbour catalogue. The choice 
of 20 neighbours has proven to keep the noise in the smooth density small while allowing one to reach a small smoothing scale (Park et al. 2007, 2008; Paper I).

As in Paper I, we define three mass bins at each redshift (two at $z=3.1$ and one at $z=4$ ) with the same number of targets, and subdivide them into 3 density bins so that each of the 9 (respectively 6 or 3 ) bins has the same number of targets. This introduces three mass and six density thresholds $M_{i}(z), \Delta_{j, i}(z) ; i \in\{0,1,2\}, j \in\{1,2\}$, that can be seen in Figure A1 of Paper I. The values of $M_{0}, M_{1}, M_{2}$ at $z=0$ are respectively $7.68 \times 10^{12}, 1.10 \times 10^{13}$, and $1.98 \times 10^{13} h^{-1} \mathrm{M}_{\odot}$.

Therefore, we stress here that the results obtained in this paper are based on a constant number density of the halo catalogue rather than fixed mass bins. In $\S 3.5$, we address the issue of the mass and density dependence using fixed bins.

\subsection{Characterising the alignment signal}

In order to detect an alignment signal for a given angle $\theta=(\boldsymbol{u}, \boldsymbol{v})$ between any two vectors $u$ and $v$ associated to a halo, we used the normalized pair counts following Brainerd (2005) and Yang et al. (2006), comparing the measured number of pairs with the expected count from the random case.

- We counted $N(\theta)$ the number of pairs for a given $\theta$

- We then randomly reorder $\boldsymbol{u} 100$ times

- We calculated the mean and standard deviation $\left\langle N^{\mathrm{R}}(\theta)\right\rangle$ and $\sigma_{\theta}$

- We considered the normalized pair count $f(\theta)=$ $\frac{N(\theta)}{\left\langle N^{\mathrm{R}}(\theta)\right\rangle}$

- The strength of the signal (error bars) is given by $\frac{\sigma_{\theta}}{\left\langle N^{\mathrm{R}}(\theta)\right\rangle}$

In three dimensions, a uniform distribution of angles yields $f(\cos \theta)=1$, therefore we used bins with constant width in $\cos \theta$. A value of $f(\cos \theta \simeq 0) \gg 1$ shows an antialignment (orthogonality), while $f(\cos \theta= \pm 1) \gg 1$ shows an alignment.

\section{RESULTS}

\subsection{Distribution of spins and shape parameters}

\subsubsection{Spin parameter}

The rotation of haloes can be quantified by the spin parameter as defined by Peebles (1969):

$$
\lambda=\frac{|\boldsymbol{J}| \sqrt{|E|}}{G M^{5 / 2}}
$$

where $\boldsymbol{J}$ is the sum of the angular momenta of each particle in the halo, and $E=W+K$ is the total (kinetic plus potential) energy ${ }^{1}$. This corresponds to the ratio of the rotation

1 We note that the potential energy $W$ is computed using the smooth potential as calculated in the simulation. The calculation of the potential may affect the calculated spin parameter (Ahn et al. 2014).

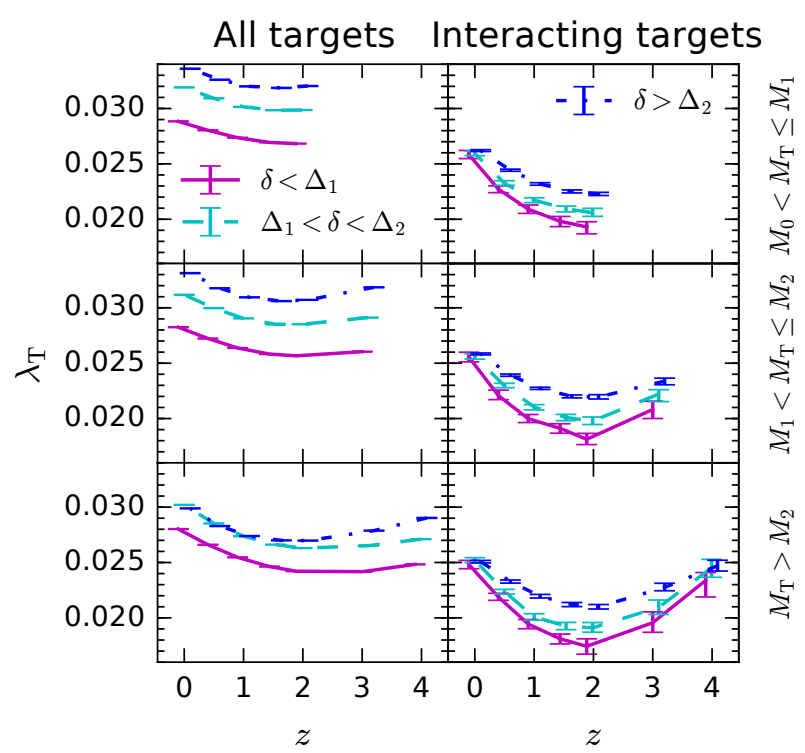

Figure 1. Spin parameter of all (left column) and interacting (right column) targets for target masses $M_{0}<M_{\mathrm{T}}<M 1$ (top), $M_{1}<M_{\mathrm{T}}<M_{2}$ (middle), and $M_{\mathrm{T}}>M_{2}$ (bottom panel), and $\delta>\Delta_{2}$ (dash-dotted lines), $\Delta_{1}<\delta<\Delta_{2}$ (dashed lines), and $\delta>\Delta_{2}$ (solid line). The lines are median values, and the errorbars show the $95 \%$ confidence intervals obtained by bootstrapping 1000 times.

to the random motions. Galaxies with purely random motion have a spin parameter of 0 , and close to unity for fully rotationally-supported galaxies.

We studied the distribution of the spin parameter $\lambda$ of interacting targets in the 9 bins of mass and density defined in $\S 2$. Figure 1 shows the median of the distribution of the spin parameter of interacting haloes for the three mass bins $M_{0}<M_{\mathrm{T}}<M_{1}$ (top), $M_{1}<M_{\mathrm{T}}<M_{2}$ (middle), and $M_{\mathrm{T}}>M_{2}$ (bottom panel), for all (left-hand column) and interacting (right-hand column) targets. In each panel, we show the median of each subsamples in the three density bins $\delta>\Delta_{2}, \Delta_{1}<\delta<\Delta_{2}$, and $\delta<\Delta_{1}$. The error bars on the median show the $95 \%$ confidence intervals obtained by bootstrapping 1000 times.

In the left-hand panels, the spin parameters of all targets first decrease until $z \simeq 2$, then increase until $z=0$. At fixed mass, targets in high-density regions have a higher median spin than the lower-density ones. However, we note that targets in the top panel have fewer than 300 particles at $z>1.5$; while targets in the middle and bottom panel have fewer than 300 particles for $z>2$. Therefore, the discreteness effect may affect the estimation of the spin (Bett et al. 2007), therefore the evolutions at $z>1.5$ (top panel) or 2 (middle and bottom panels) may not be reliable. For haloes more resolved than 300 particles, the median value of the spin is increasing with decreasing redshift. The typical increase between $z=2$ and 0 is $7 \%$ in the low-mass bin, and up to $15 \%$ in the high-mass and low-density bin.

The very weak evolution of the spin with mass is consistent with previous studies, that did not find any dependence of the spin parameter distribution on mass (Bett et al. 2007). However, the very large volume and good statistics of HR4 enabled us to detect a weak but significant signal of 


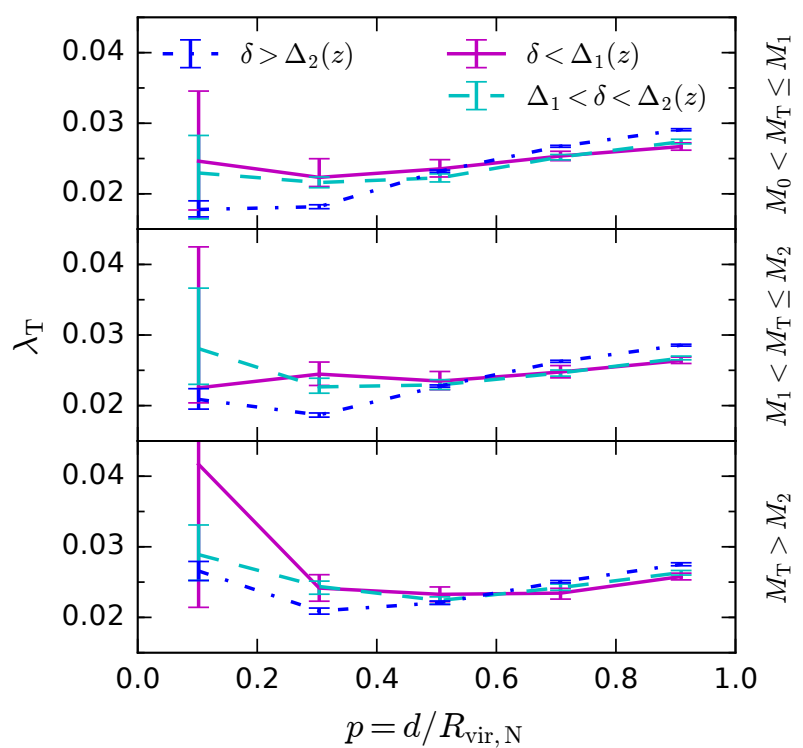

Figure 2. Spin parameter of interacting targets (at $z=0$ ) as a function of the normalized separation $p=d / R_{\mathrm{vir}}$.

the influence of interactions in the distribution of the spin parameter. In the right-hand panels, the spin parameter of interacting targets shows a similar behaviour to that of all targets: It also reaches a minimum at $z \simeq 2$, and increase until $z=0$ where it interestingly has a similar value at all bin of mass and density, $\lambda_{\mathrm{T}} \simeq 0.025$. Here again, we cannot conclude about the upturn since haloes are poorly resolved at $z>2$. At $z=2$, in the higher mass bin, the median varies from 0.0175 in the low-density regions to 0.021 in high-density. The typical increase of $\lambda_{\mathrm{T}}$ of interacting targets is larger than that of all targets. Between $z=2$ and 0 , it increases by 17 to $19 \%$ in high-density regions, 26 to $32 \%$ in intermediate regions, and 33 to $42 \%$ in low-density regions.

The spin parameter of interacting target is smaller than that of all targets. This can be understood by the tidal forces from the neighbour they are interacting with. This can be seen in Figure 2, which shows the spin parameter of interacting targets at redshift 0 as a function of the separation normalized by the virial radius of the neighbour $p=d / R_{\mathrm{vir}}$. The spin parameter decreases with decreasing separation, from about 0.025 at $p=1$ to about 0.02 at $p \simeq 0.25$, which supports the idea that interactions tend to slow down the targets, since the spin parameter is smaller at smaller separation, where the tidal forces are stronger. At very-low $p<0.3$, there seems to be an upturn in the spin parameter. This effect is not significant, given the large error-bars, except in the higher mass bin, but it seems to be systematic. Interestingly, Cervantes-Sodi et al. (2012), who used the same definition of neighbours as ours, found similar results from observations while measuring the spin of spiral galaxies which have an early-type neighbour.

According to the TTT, the two-point spin correlations show an increasing correlation with a smaller separation (see eq. 16 and Figure 11 in Porciani et al. (2002)). However, this trend is lessened at lower redshifts, mainly due to the effects of nonlinear evolution. This may indicate that the positive proximity effect on the target's spin amplitude comes from the tidal interactions with the neighbour halo, which is stronger as the separation becomes smaller.

To our knowledge, this is the first numerical study of the effects of interactions on the spin of dark matter haloes.

\subsubsection{Shape parameters}

Haloes are usually not spherically symmetric. To quantify their triaxiality, we computed the pseudo-inertia tensor I defined as

$$
I_{i j}=\sum_{\alpha} x_{\alpha, i} x_{\alpha, j},
$$

where $x_{\alpha, i}$ is the $i$ th component of the relative position of particle $\alpha$ with respect to the centre of mass of the halo. This tensor has three positive eigenvalues $a^{2} \geq b^{2} \geq c^{2}$, and the corresponding three eigenvectors $\boldsymbol{a}, \boldsymbol{b}, \boldsymbol{c}$, respectively referred to as the major, intermediate, and minor axes. The choice of this tensor, rather than the reduced inertia tensor (where the contribution of each particle is weighted by $\left.1 / r^{2}\right)$ is motivated by the fact that it is easier to compare to observation, and more relevant to weak lensing studies (Bett 2012). Moreover, since PSB subhaloes do not include the mass of their own subhaloes, the inertia is not contaminated by the latter.

The effects of the weighting scheme on the results is discussed in $\S \mathrm{A}$.

The sphericity $s$, oblateness $q$, and prolateness $p$ parameters are defined as

$$
s=\frac{c}{a}, q=\frac{b}{a}, \text { and } p=\frac{c}{b} .
$$

Haloes are said to be respectively spherical, oblate, or prolate, if

$$
\begin{array}{ll}
a \simeq b \simeq c, & \text { or } s \simeq 1, \\
a \simeq b \gg c, & \text { or } q \simeq 1 \text { and } s \ll q, \\
a \gg b \simeq c, & \text { or } p \simeq 1 \text { and } q \ll 1,
\end{array}
$$

and triaxial in other cases.

Figure $3 \mathrm{a}$ and $\mathrm{b}$ respectively show the median value of the sphericity and oblateness parameters of all (left-hand columns) and interacting (right-hand columns) targets as a function of redshift, for the same bins of mass and density as Fig. 1. In the all-target case (left-hand column), the sphericity increases with decreasing redshift at all bin of mass and density, from about 0.5 to 0.55 : haloes become more spherical at lower redshifts. The oblateness increases from $z=4$ to 2 , then decreases until $z=1$, and increases again until $z=0$. At fixed redshifts and mass, for the all-targets case, $q$ and $s$ increase with density. Finally, at fixed density and redshift, the sphericity is independent of mass in the mass range probed by our constant number density bins.

These results are in agreement with previous results, for instance, Schneider et al. (2012) who studied the distributions of $s$ and $q$ in the Millennium I and II simulations (Springel et al. 2005; Boylan-Kolchin et al. 2009). However, the very good statistics of the HR4 enables us to see an additional dependence on the large-scale density.

The right-hand panels of Figs. 3a and b show the evolution of the sphericity and oblateness parameters of those target that are undergoing an interaction. The sphericity 


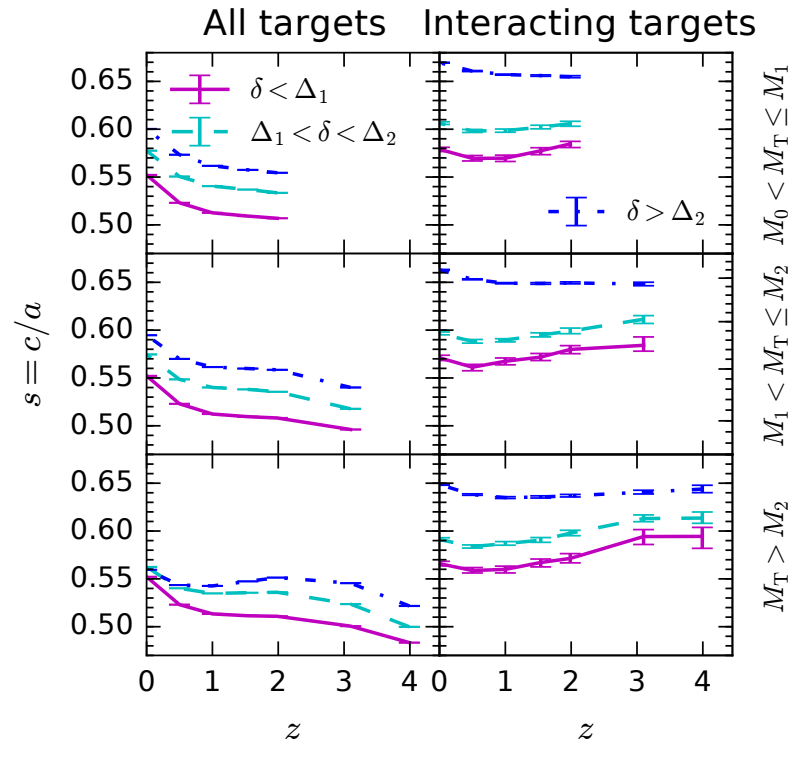

(a) Sphericity $s=c / a$

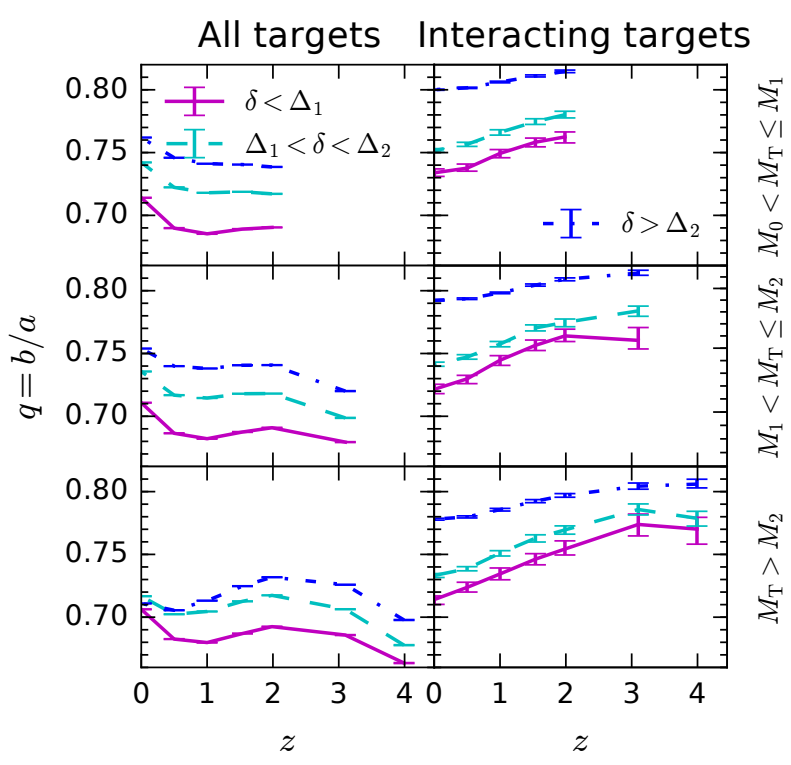

(b) Oblateness $q=b / a$

Figure 3. Sphericity (left) and oblateness (right) of interacting targets; same legend as Fig. 1.

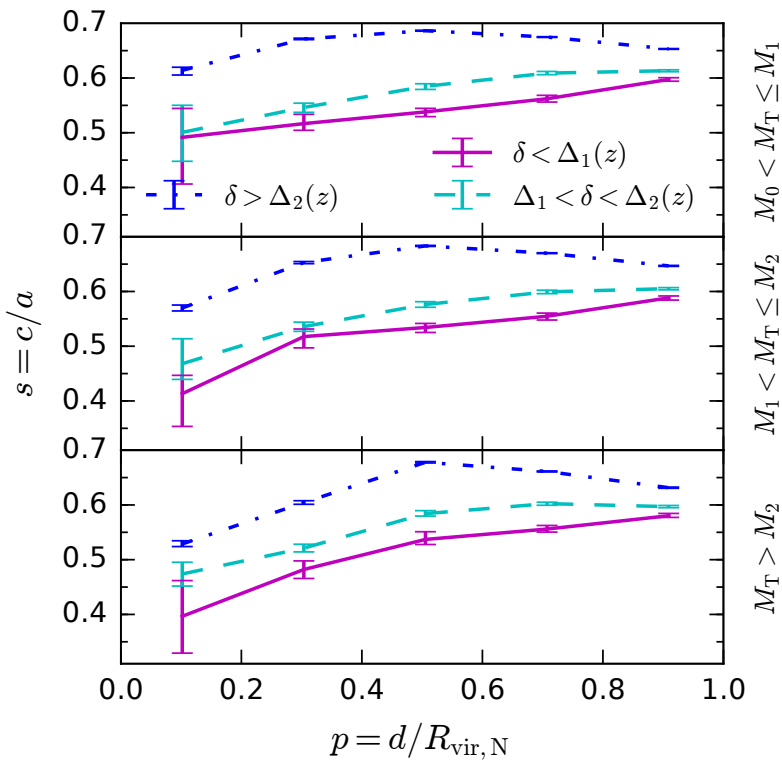

(a) Sphericity $s=c / a$

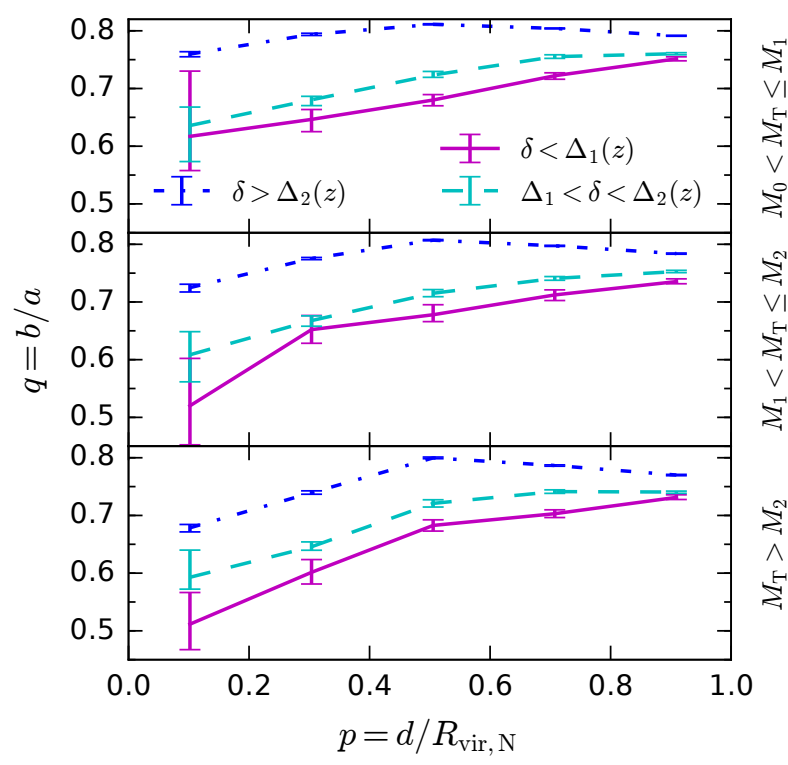

(b) Oblateness $q=b / a$

Figure 4. Sphericity and oblateness of interacting targets as a function of the normalized separation $p=d / R_{\mathrm{vir}}$ at $z=0$; same legend as Fig. 1.

and oblateness of interacting targets are higher than all targets: both are significantly higher for the interacting targets. Moreover, their redshift-evolution is different: both decrease with decreasing redshift, as opposed to the all-target case.

The dependence of $s$ and $q$ on the normalized separation $p=d / R_{\text {vir }}$ at $z=0$ is shown on Fig. $4 \mathrm{a}$ and $\mathrm{b}$. In both cases, the parameter decreases with decreasing separation. However, it is interesting to notice that in high- and intermediate densities, the parameter reaches a maximum, respectively at $p \simeq 0.5$ and 0.7 , while at low-density, the decrease is monotonous. In the latter case, the maximum is presumably located outside of the virial radius of the neighbour.

This is surprising at first, but can be understood by these two facts: (1) the number of interactions decreases with $p$, so we are dominated by large- $p$ interactions (loose-pairs), which tend to have a larger sphericity and oblateness. (2) In the high-density bin, the maximum $s$ and $q$ are reached at $p \simeq 0.5$, then both quantities decrease. In the intermediatedensity bin, the maximum seems to be reached at $p \simeq 0.9$. It is reasonable to believe that for the lower bin of density, similar behaviour can be seen beyond $R_{\mathrm{vir}}$. Therefore, non- 


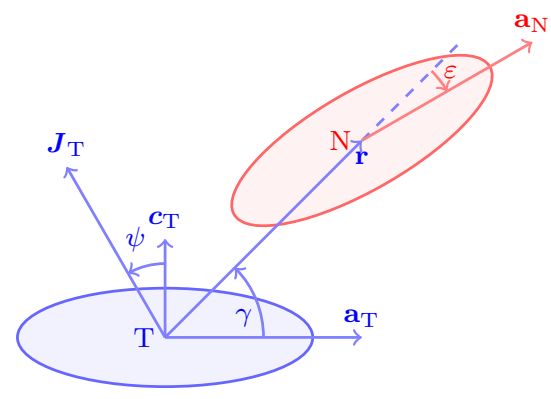

Figure 5. Definition of the angles $\gamma=\left(\boldsymbol{a}_{\mathrm{T}}, \boldsymbol{r}\right), \varepsilon=\left(\boldsymbol{a}_{\mathrm{N}}, \boldsymbol{r}\right)$, and $\psi=\left(\boldsymbol{c}_{\mathrm{T}}, \boldsymbol{J}_{\mathrm{T}}\right)$.

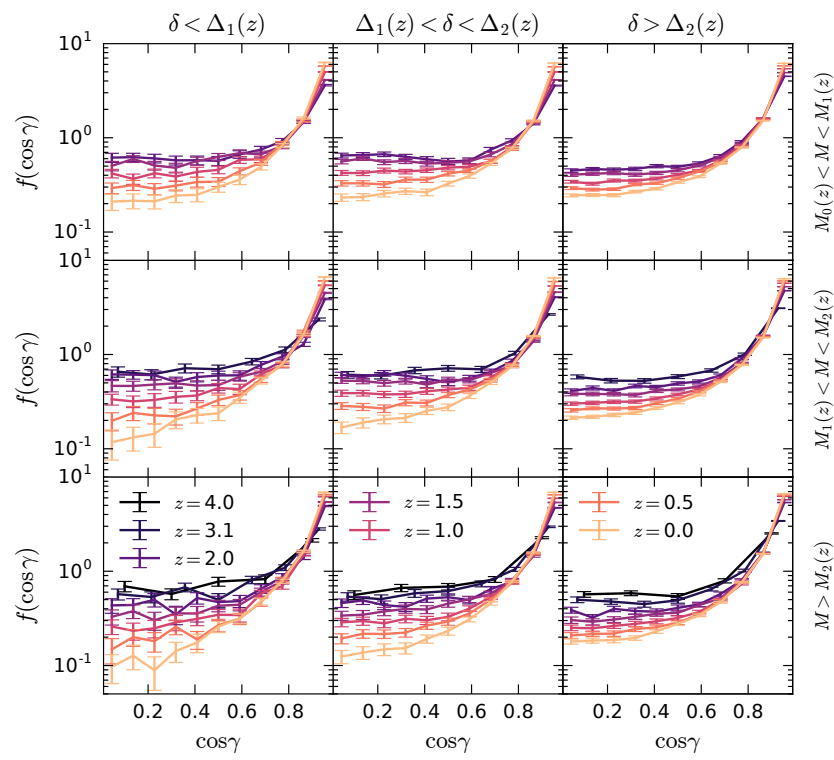

Figure 6. Time evolution of the distribution of $\cos \gamma$, where $\gamma=$ $\left(\boldsymbol{a}_{\mathrm{T}}, \boldsymbol{r}\right)$ is the direction of the nearest neighbour halo relative to the major axis of the target. The three rows correspond to the three mass bins as in 1 , while the three columns correspond to the three density bins. The error-bars are obtained by shuffling the pairs (c.f. § 2.3 ).

interacting targets, which are located at more than $1 R_{\text {vir }}$ from the nearest neighbour, must have a lower $s$ and $q$.

Halo shapes $(c / a$ and $b / a)$ as shown in Figure 4 become more elongated for closer pairs, which may come from stronger tidal interactions (their shapes are stretched toward each other). This trend becomes less obvious in denser regions, where the interaction with the closest neighbour becomes less important with respect to interactions with other neighbouring halos. The effects from other halos may thus distort the pair tidal interactions.

\subsection{Alignment of the principal axes}

We studied the alignment of the major axis $\boldsymbol{a}$ associated with the largest eigenvalue $a$ of the target and neighbour. In the following, we study the angles $\gamma=\left(\boldsymbol{a}_{\mathrm{T}}, \boldsymbol{r}\right)$ and $\varepsilon=\left(\boldsymbol{a}_{\mathrm{N}}, \boldsymbol{r}\right)$, where $\boldsymbol{r}$ is the position vector of the neighbour with respect to the target (see Fig. 5). Note that these two angles are defined modulo $\pi / 2$, since the direction of the major axis

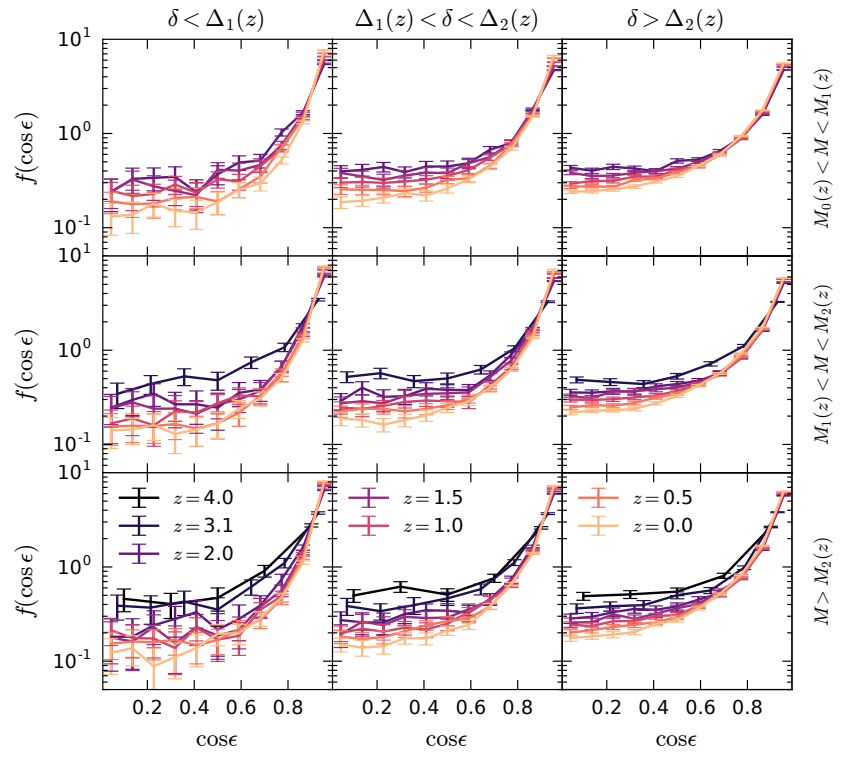

Figure 7. Time evolution of the distribution of $\cos \varepsilon$, where $\varepsilon=$ $\left(a_{\mathbf{N}}, \boldsymbol{r}\right)$ is the angle between the halo separation vector and the major axis of the nearest neighbour halo. The legends are the same as Fig. 6.

is irrelevant, therefore we study the distribution of $|\cos \gamma|$ and $|\cos \varepsilon|$ between 0 and 1. Moreover, in order to remove noise from poorly-defined shapes, we require the oblateness parameter to be smaller than 0.8 , so that the major axis is well-defined. This effectively excludes spherical and oblate haloes. Again, in the lower mass bin, haloes have more than 300 particles from $z=2$, and from $z=1.5$ in the intermediate and higher mass bins.

Fig. 6 and 7 respectively show the normalized pair counts, as defined in $\S 2.3$, for $|\cos \gamma|$ and $|\cos \varepsilon|$ in the nine bins of mass and density previously defined. The error-bars were obtain following the method described in $\S 2.3$. Both figures show very similar features. Note however that, even though the definitions of the angles $\gamma$ and $\varepsilon$ are symmetric, the targets and neighbours belong to different catalogues, so we do not necessarily expect the same behaviour. Namely, for a given target $\mathrm{T}$, the neighbour of its neighbour $\mathrm{N}$ is not necessarily $\mathrm{T}$. There is a clear alignment signal at all redshifts, masses, and densities, meaning that the neighbour is preferentially located in the direction of the major axis of the target, and that the main axis of the neighbour is well aligned with the direction to the target. At fixed mass, the signal is more significant at higher densities, since the statistics are better. However, the alignment itself seems to be stronger at low densities. Given the small range of mass probed here, no dependency can be seen. The mass and density dependency will be studied in more detail in $\S 3.5$. The signal is weaker at higher redshifts, and becomes stronger as time passes. In addition, the alignment signal evolves more in low-density regions than in high density.

We also studied the alignment between the minor axis of the target and its spin, as defined by the angle $\psi=\left(\boldsymbol{c}_{\mathrm{T}}, \boldsymbol{J}_{\mathrm{T}}\right)$. Similarly to the major axis case, in order to ensure that the minor axis is well-defined, we require the prolateness to be smaller than 0.8. Figure 8 shows the normalized pair count for $\psi$ for the previously-defined mass and density bins. 


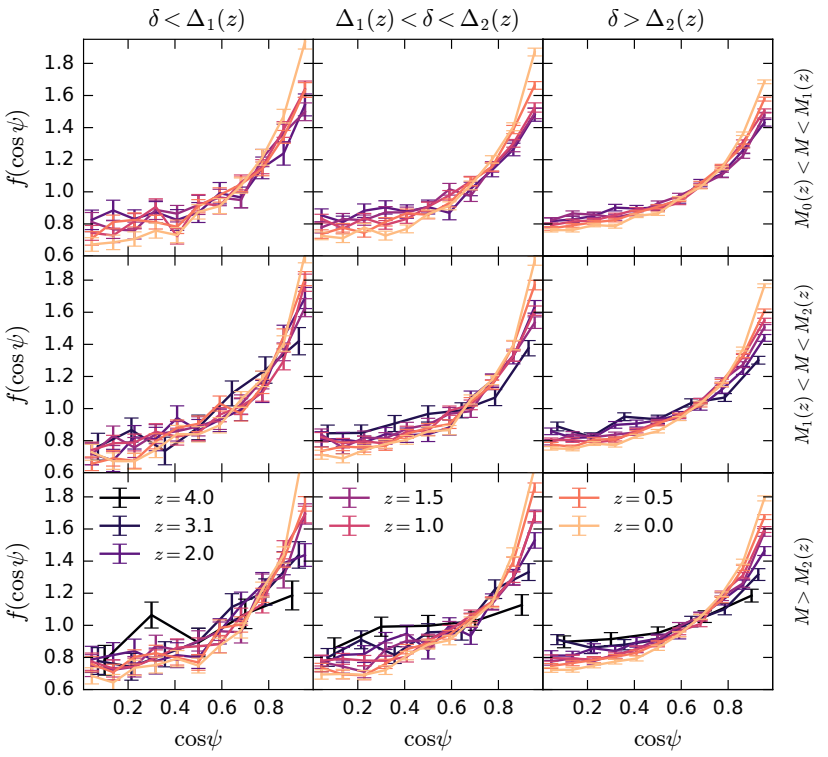

Figure 8. Time evolution of the distribution of $\cos \psi$, where $\psi=$ $\left(\boldsymbol{c}_{\mathbf{T}}, \boldsymbol{J}_{\mathbf{T}}\right)$ is the angle between the minor axis of the target halo and its angular momentum. The legends are the same as Fig. 6.

The minor axis and spin are well aligned, as was previously found by Aragón-Calvo et al. (2007). The alignment signal increases with mass, and decreasing redshift and density. Again, it is interesting to see that it is present at redshifts as high as 4 .

To summarise, the position and the major axis of the neighbour are aligned with the major axis of the target, constraining the interaction within a small solid angle with respect to the major axis of the target. These results are in good qualitative agreement with observations (Yang et al. 2006; Wang et al. 2008), who found that the distributions of satellites is aligned with the major axis of the central galaxy.

In order to illustrate how prolate and triaxial pairs interact, we proceeded as follows. We first considered pairs such as both $q_{\mathrm{N}}$ and $q_{\mathrm{T}}<0.8$. This ensures that the major axis of both members are well-defined. This represents 4212799 (2 383237$)$ pairs, or $29.5 \%$ (28.1\%) of interacting pairs at redshift $0(1)$.

Figure 9 illustrates the distribution of the positions and the orientations of the major axis of the neighbours with respect to the target. We divided the sample of neighbours into three equal subsamples according to their separation normalized to the virial radius of the neighbour $p=d / R_{\mathrm{vir}, \mathrm{N}}$, and show the $33 \%$ closest pairs $(0<p<0.66$, blue $)$ and $33 \%$ further apart pairs $(0.85<p<1$, red). Neighbours are randomly drawn at angles $\gamma$ following the acceptance-rejection method: for each bin of $\gamma$, we drew a uniformly-distributed random number and compared to the normalized distribution of $\gamma$. If the random number is smaller than the probability density of $\gamma$, we accept it and draw a neighbour at this $\gamma$. Since $\gamma$ is defined between 0 and $90^{\circ}$, we populated the 4 quadrants by drawing four independent random realisations of the positions. It clearly appears that the neighbours are preferentially located at low latitudes with respect to the major axis of the target $\left(\gamma \simeq 0\right.$ or $\left.180^{\circ}\right)$. At a given $\gamma$, we randomly picked a neighbour falling into this bin, and placed it at its normalized separation $p$ and orientate its major axis with an angle $\varepsilon$ from the line connecting it to the target. As expected from Fig. 7, the major axis of the neighbour is on average aligned with the line connecting it to the target.

This is confirmed in Fig. 10, which shows the distribution of $\cos \varepsilon$ for fixed ranges of gamma: $0 \leq \gamma<1.8^{\circ}$ (blue), $43.2^{\circ} \leq \gamma<45^{\circ}$ (red), and $88.2^{\circ} \leq \gamma<90^{\circ}$ (green), for close $(0<p<0.66$, left $)$ and distant $(0.85<p<1$, right $)$ interactions, and at redshifts 0 (top) and 1 (bottom). At all epochs and normalized distance $p$, there is a strong alignment signal in $\cos \varepsilon$. For $0.85 \leq p<1$ (loose pairs), the distribution of $\cos \varepsilon$ is independent of the position angle $\gamma$. However, for $0 \leq p<0.66$ (close pairs), the distribution of $\cos \varepsilon$ depends on $\gamma$. For $\gamma \simeq 45-90^{\circ}$, the distribution of $\cos \varepsilon$ is similar to that of the loose pairs. However, at small $\gamma$, the alignment signal is stronger. This shows that the alignment of the major axes is given by the large-scale structure, since it is present at all $p$, and the influence of the neighbours increases this alignment signal at small separations. Fig. 10 shows that the major axis of the neighbour halo is pointing toward the target halo in both loose and close pair cases. On the other hand, the degree of alignment is independent of the relative angular position of the neighbour halo in the case of loose pairs, but depends on it in the close pair case. We interpret this latter phenomenon due to the tidal force of the target halo on the close neighbour. We attribute the alignment of loose pairs, which is independent of the internal property of the target halo, to the tidal shear force set up by the large-sale mass distribution.

\subsection{Spin alignments}

In this section, we study the alignment of the spin of the target haloes with respect to their neighbours. We define the angle $\alpha=\left(\boldsymbol{J}_{\mathrm{T}}, \boldsymbol{r}_{\mathrm{N}}\right)$ between the spin of the target and the direction to the nearest neighbour, and $\phi=\left(\boldsymbol{J}_{\mathrm{T}}, \boldsymbol{J}_{\mathrm{N}}\right)$ the angle between the spins of the target and neighbour (see Fig. 11). Since spins have a direction, the angles are considered between 0 and $180^{\circ}$.

Figure 12 shows the alignment between the spins of the target and neighbour. At $z=4$, there is a slight preference for an anti-parallel configuration $(\cos \phi \simeq-1)$ in all environment, which is stronger for lower densities. At $z=3.1$, the situation is similar at low- and intermediate densities, but starts to change in high-density regions, where the distribution becomes more symmetric, and the parallel $(\cos \phi \simeq 1)$ and anti-parallel $(\cos \phi \simeq-1)$ configurations are of the same order. At $z=2$, the parallel configuration is dominant in high-density regions, while the situation is becoming symmetric in the lower density bins. Eventually, at all mass and density, by $z=0$, the alignment signal for the parallel configuration becomes stronger and stronger and the anti-parallel configuration is almost lost. Interestingly, the alignment signal is stronger at lower density, where the initial configuration was mostly anti-parallel. Similar results to those from Fig. 8 can be achieved for $\cos \alpha$, we omitted them for the sake of clarity.

We can illustrate the alignment of spin as Fig. 9. This time, we do not need to restrict ourselves to prolate pairs, and consider all interacting pairs. However, since the alignment signal is weaker for the spins, a figure similar to Fig 9 is not as instructive as before. 

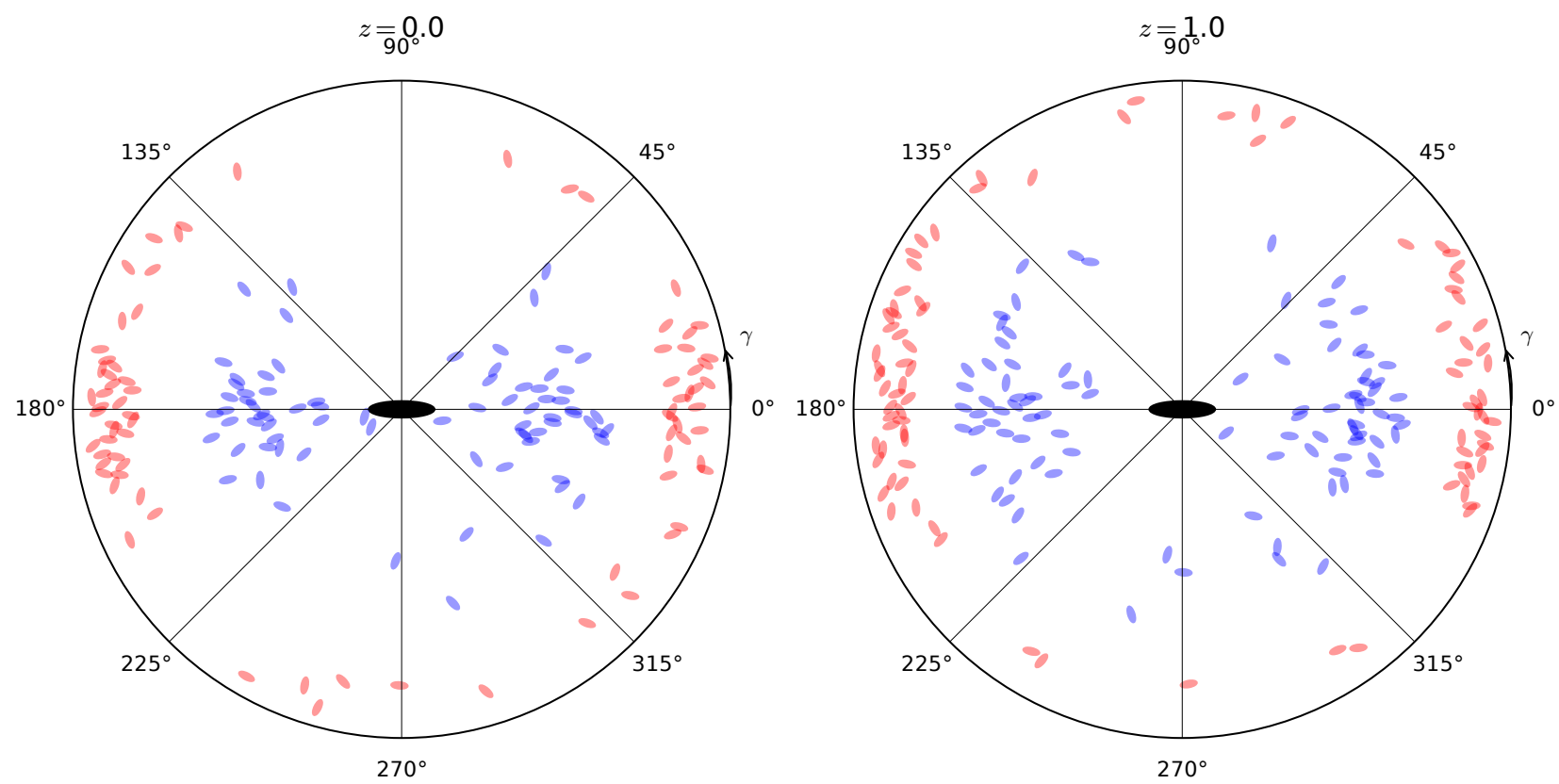

Figure 9. Distribution of the angular location and orientation of the nearest neighbour with respect to the target. The black ellipse at the centre represents the target. Neighbours are placed at positions $\gamma$ according to the probability distribution of $\gamma$. Note that the neighbours are not necessarily smaller than the target halo. The blue and red ellipses represent the neighbours with their orientation with respect to the line connecting it to the target. The blue (red) ellipses show the $33 \%$ closest pairs $(0<p<0.66)$, and the red ones the $33 \%$ further apart pairs $(0.85<p<1)$. For a given $\gamma$, the orientation of the neighbour $\varepsilon$ and the normalized separation $p$ are chosen randomly among all neighbours at this $\gamma$.

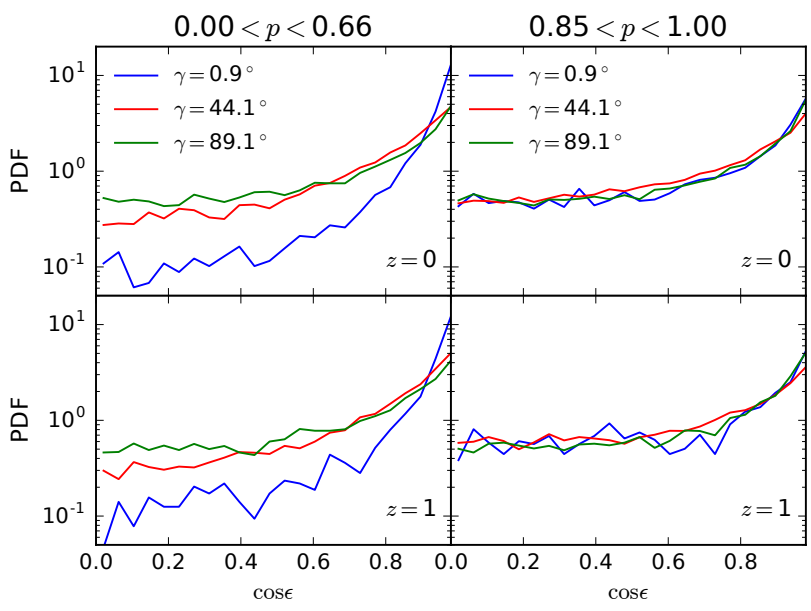

Figure 10. Distribution of $\cos \varepsilon$ for $0 \leq \gamma<1.8^{\circ}$ (blue), $43.2^{\circ} \leq$ $\gamma<45^{\circ}$ (red), and $88.2^{\circ} \leq \gamma \leq 90^{\circ}$ (green). The left-hands panels show close pairs with normalized separation $0<p=d / R_{\mathrm{vir}, \mathrm{N}}<$ 0.66 , and the right-hand panels loose pairs with $0.85<p<1$. The top and bottom rows respectively show the results at redshifts 0 and 1 .

Instead, we show in Fig. 13 the distribution of $\cos \phi$, the angle between the halo spins in three different ranges of $\alpha$. We divide the sample of pairs into three equal subsamples according to their normalized separation $p$. We find that the spin-spin alignment changes sensitively depending on the relative angular position of the nighbour with respect to the spin of the target. For neighbours located at high lati-

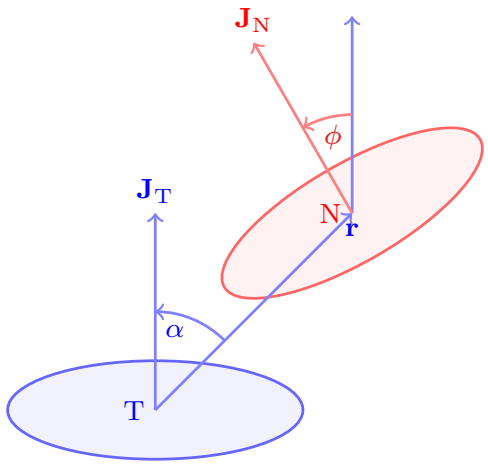

Figure 11. Definition of the angles $\alpha$ between the spin of the target $\boldsymbol{J}_{\mathrm{T}}$ and the direction of the neighbour $\boldsymbol{r}_{\mathrm{N}}$, and the angle $\phi$ between $\boldsymbol{J}_{\mathrm{T}}$ and the spin of the neighbour $\boldsymbol{J}_{\mathrm{N}}$.

tudes $\left(\alpha \simeq 0\right.$ or $\left.180^{\circ}\right)$, the spins tend to be orthogonal, while at equatorial latitudes $\left(\alpha \simeq 90^{\circ}\right), \cos \phi$ has a bimodal distribution peaking at $\cos \phi= \pm 1$, corresponding to parallel and anti-parallel cases. In all cases, the distribution of $\cos \phi$ is skewed towards positive alignment $(\langle\cos \phi\rangle>0)$. We saw in Fig. 8 and 6 that the equatorial configuration is preferred, which explains the larger fluctuations at 1.8 and $178.2^{\circ}$ in Fig. 13. The preference for $\alpha \simeq 90^{\circ}$ is also responsible for the apparent bimodal distribution of $\cos \phi$ in Fig. 12: neighbours located in the equatorial plane tend to have their spin either parallel or anti-parallel to the target (red lines), while those located in polar directions have their spin orthogonal, but are less common and thus only weekly affect the distri- 


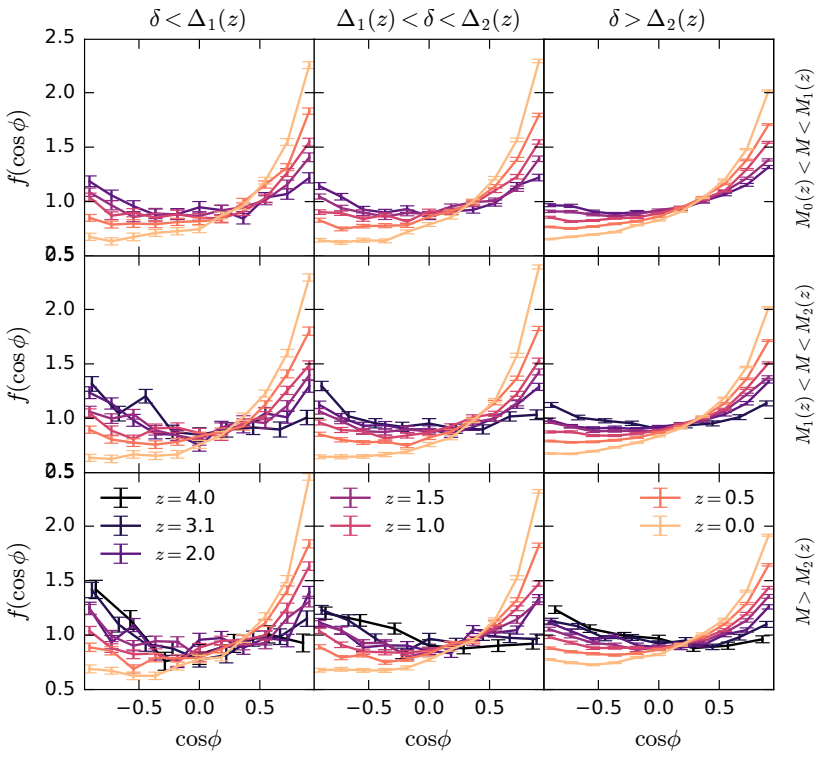

Figure 12. Time evolution of the distribution of $\cos \phi$, where $\phi=\left(\boldsymbol{J}_{\mathbf{T}}, \boldsymbol{J}_{\mathbf{N}}\right)$ is the angle between the spin of the target and that of the neighbour. The legend is the same as Fig. 6.

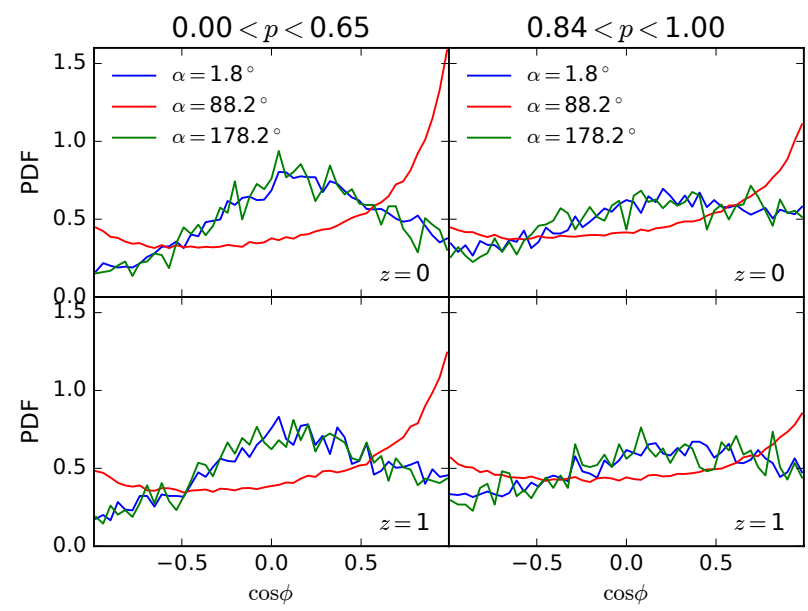

Figure 13. Distribution of $\cos \phi$ for $0 \leq \alpha<3.6^{\circ}$ (blue), $86.4^{\circ} \leq$ $\alpha<90^{\circ}$ (red), and $176.4^{\circ} \leq \alpha \leq 180^{\circ}$ (green). The left panels are for close interaction with normalized halo separation for the $33 \%$ closest pairs $0<p=d / R_{\mathrm{vir}, \mathrm{N}}<0.65$, and the right panel for the $33 \%$ furthest apart pairs $(0.84<p<1)$. The top row is for redshift 0 and the bottom row at redshift 1 .

bution of $\cos \phi$. Similarly to Fig. 10, the alignment signal is affected by the normalized separation: it is slightly weaker in the loose pairs, while close interactions tend to have a stronger interaction signal. However, the effect of the distance on the alignment is not as strong as in Fig. 10. We checked that the stronger alignment in Fig. 10 is not due to the the selection criteria for oblate haloes $(q<0.8)$ by applying the same criterion in Fig. 13. The stronger alignment of the shapes is thus a real effect, and can be understood as follows: The initial alignment of the spins and the shapes with the large-scale structure comes from the tidal field (TTT). However, as structure evolve non-linearly through merger

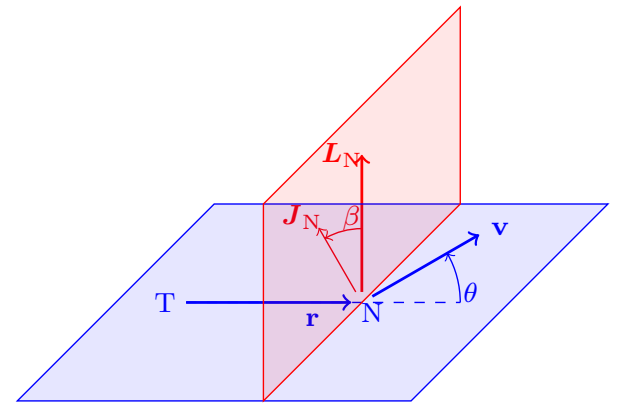

Figure 14. Definition of the angles $\theta=(\boldsymbol{r}, \boldsymbol{v})$ and $\beta=\left(\boldsymbol{L}_{\mathrm{N}}, \boldsymbol{J}_{\mathrm{N}}\right)$, where $\boldsymbol{L}_{\mathrm{N}}=\boldsymbol{r} \times \boldsymbol{v}$.

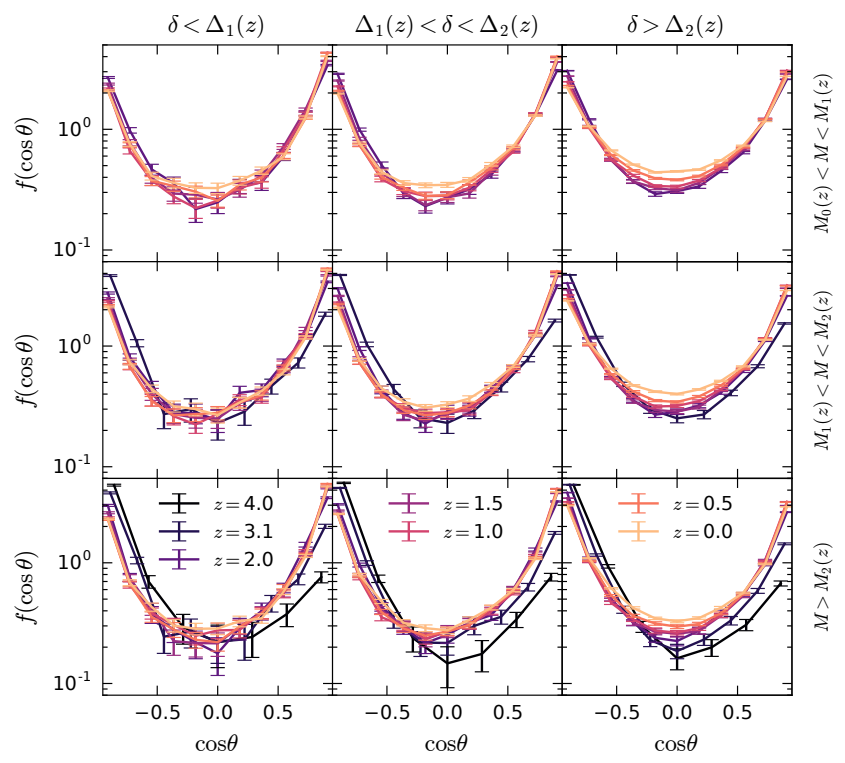

Figure 15. Distribution of $\cos \theta$, where $\theta=(\boldsymbol{r}, \boldsymbol{v})$ is the angle between the velocity and the position of the neighbour in the frame of the target halo, same legend as Fig. 6.

events, the alignment becomes more complex: merger can completely flip the spins (Porciani et al. 2002), while the shapes are more robust. Therefore, it is not surprising that the alignment is stronger in the case of the shapes.

\subsection{Orbits of the interactions}

\subsubsection{Incident angle}

In this section, we study the distribution of the angle $\theta=$ $(\boldsymbol{r}, \boldsymbol{v})$ between the position and velocity of the neighbour in the frame of the target (see Fig. 14). A value of $\cos \theta \simeq(-) 1$ means that the satellite is radially receding from (approaching) the target, while tangential orbits have $\cos \theta \simeq 0$.

Figure 15 shows the normalized pair count of $\cos \theta$ for the same ranges of density and target mass as in the previous section. At all masses, densities, and redshifts, there is a clear alignment signal: the orbits are preferentially radial. At early times, $z \geq 3$, there is a peak at $\cos \theta=-1$, corresponding to radially infalling orbits. The peak decreases with decreasing redshift, and by $z=2$ the distribution of angles becomes more symmetric and the number of radially 


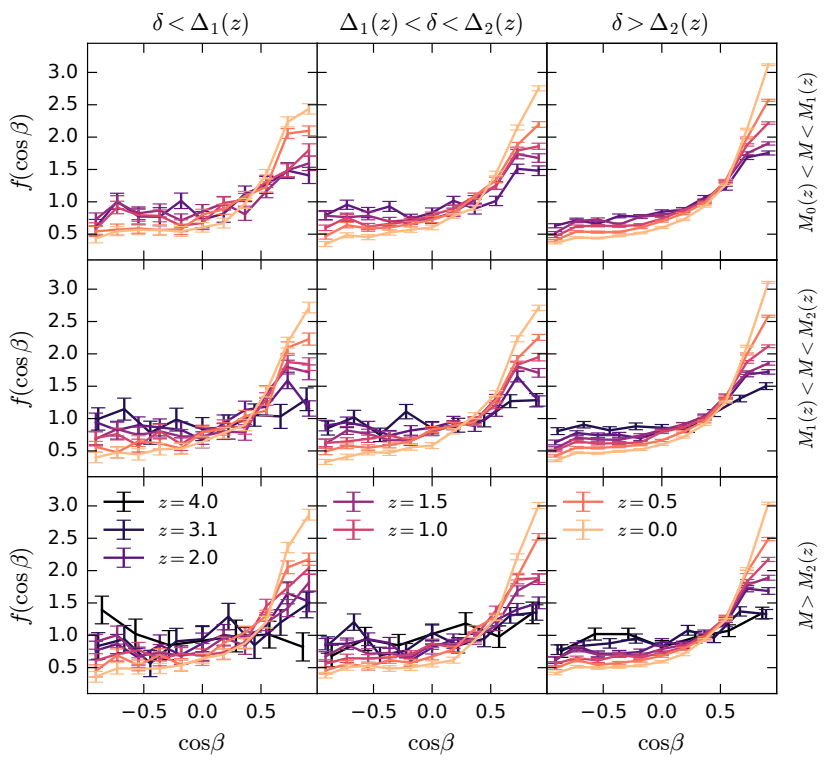

Figure 16. Distribution of $\cos \beta$, where $\beta=\left(\boldsymbol{L}_{\mathrm{N}}, \boldsymbol{J}_{\mathrm{N}}\right)$ is the angle between orbital angular momentum of the target and $\boldsymbol{J}_{\mathrm{N}}$ its spin.

receding orbits increases. Meanwhile, the contribution of orbits with $|\cos \theta| \ll 1$, corresponding to tangential orbits, increases. This effect was already seen in Paper I, where we separated the orbits according to their position in the $(p, \xi)$ plane $^{2}$, where $p$ is the pair separation in units of virial radius of the neighbour, and $\xi$ is the mass ratio. We showed that the typical trajectory of the orbit is radial at the first encounter, then becomes more random.

The effects of mass and density on the normalized pair count are weak. At low density, the radially receding orbits start to dominate at $z=2$, while at intermediate densities, they become dominant at $z=1.5$. In the higher-density bin, they become dominant at $z=1$, except in the higher mass bin where radially receding orbits are the same order as radial infalling.

\subsubsection{Prograde versus retrograde encounters}

Finally, we looked at the angle $\beta=\left(\boldsymbol{L}_{\mathrm{N}}, \boldsymbol{J}_{\mathrm{N}}\right)$ between the spin of the neighbour and its orbital angular momentum $\boldsymbol{L}_{\mathrm{N}}=\boldsymbol{r} \times \boldsymbol{v}$. A value of $\cos \beta=(-) 1$ means that the orbit is prograde (retrograde), while $\cos \beta=0$ either means a radial orbit $\left(\boldsymbol{L}_{\mathrm{N}}=0\right)$ or an orbit in the plane of rotation of the neighbour $\left(\boldsymbol{L}_{\mathrm{N}} / / \boldsymbol{J}_{\mathrm{N}}\right)$. Therefore, we restricted ourselves to cases where $|\cos \theta|<0.5$, ensuring a non-radial orbit.

Figure 16 shows the normalized pair count for $\cos \beta$. At $z=4$, the alignment signal is consistent with no alignment (i.e. random orbits) for low- and intermediate densities, while at high-densities, there is a slight excess of prograde orbits $(\cos \beta \simeq 1)$, and depletion of retrograde orbits $(\cos \beta \simeq-1)$. At lower redshifts, the excess of prograde encounters starts to develop at lower densities and at all masses too. Overall, the alignment signal is the strongest at high

2 In Paper I, the mass ratio was denoted as $q$. However here, $q$ was already used for the oblateness.

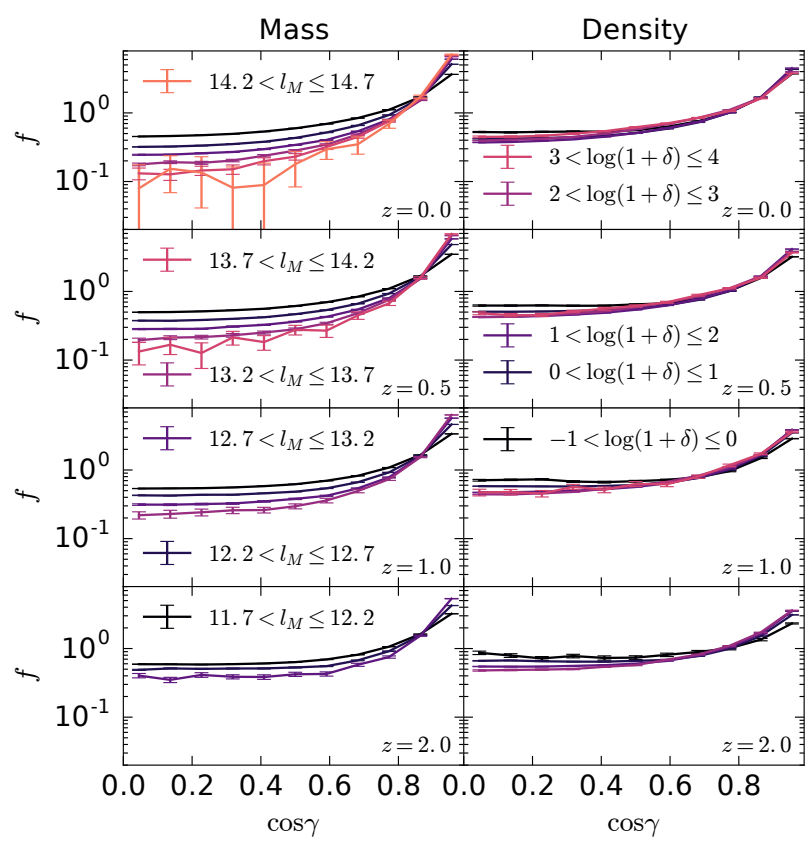

Figure 17. Effect of mass and density on the distribution of $\cos \gamma$, where $\gamma=\left(\boldsymbol{a}_{\mathrm{T}}, \boldsymbol{r}\right)$ is the direction of the nearest neighbour halo relative to the major axis of the target. Left: effect of mass, using six bins with $\Delta l_{m}=0.5$, and $l_{M}=\log _{10}\left(h M / M_{\odot}\right)$. Right: effect of the large-scale density, using five density bins with $\Delta \log _{10}(1+$ $\delta)=1$.

density, while the effects of mass in the range probed by the constant-number density bins is very weak.

\subsection{Dependence on mass and density}

In this section, we study the dependence of the alignment on mass and density. We focus on the angles $\gamma=\left(\boldsymbol{a}_{\mathrm{T}}, \boldsymbol{r}\right)$, and $\phi=\left(\boldsymbol{J}_{\mathrm{T}}, \boldsymbol{J}_{\mathrm{N}}\right)$. In the previous section, we kept a constant number density of targets in order to study the redshift evolution, which limited the sample at low redshift. In this section, we take advantage of the whole range of mass and density. We focus on the alignment between the position angle of the neighbour and the orientation (major axis) of the target.

Fig. 17 and 18 respectively show the normalized pair counts, as defined in $\S 2.3$,for $|\cos \gamma|$ and $\cos \phi$ in 6 bins of mass (left column) and 5 bins of density (right-hand panels), equally spaced in log-space. Both angles show a strong alignment. As for $\cos \gamma$, the alignment increase with redshift and mass, but the dependence on the large-scale density is very weak, especially at high-redshift. $\cos \phi$ shows a more complex alignment, where the alignment is weaker at low (dark) and high (bright) mass and density, and stronger at intermediate mass and densities. The maximum is reached for $1<1+\delta<10$ at every redshift, The lower alignment in low density is consistent with what is seen for other quantities, and presumably due to weaker tidal fields. At high density, multiple interactions with neighbours will flip the spins and weaken the alignment, while this does not affect the shapes as much. The mass dependency is also weaker than in the shape alignment. Fig. 19 shows the alignment 


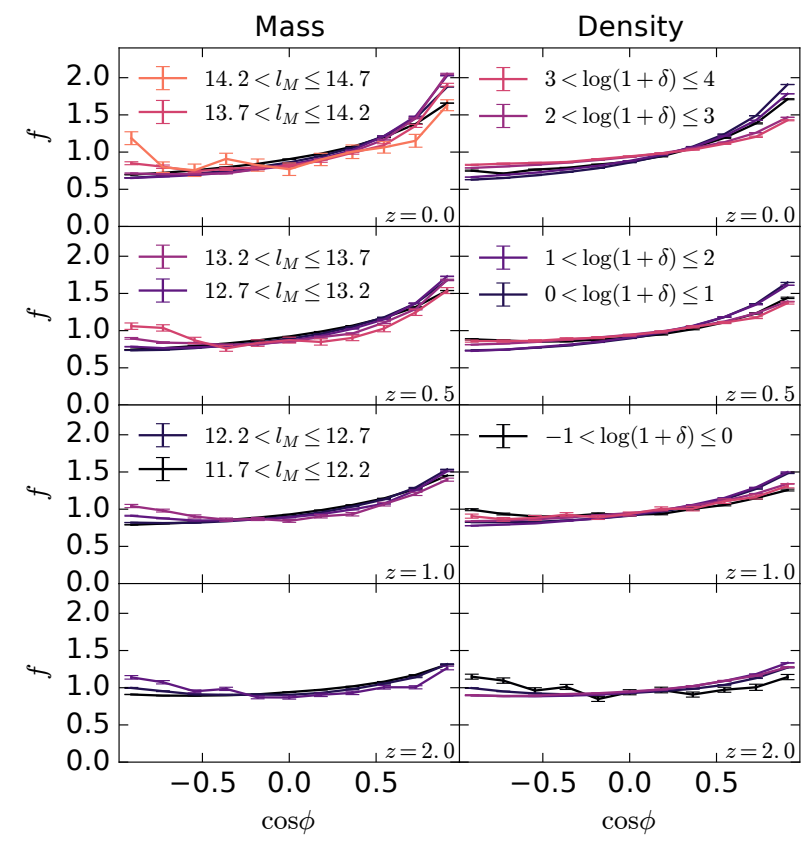

Figure 18. Effect of mass and density on the alignment of the spins of interacting halos, as shown by the distribution of $\cos \phi$, where $\phi=\left(\boldsymbol{J}_{\mathrm{T}}, \boldsymbol{J}_{\mathrm{N}}\right)$. Same legend as Fig. 17

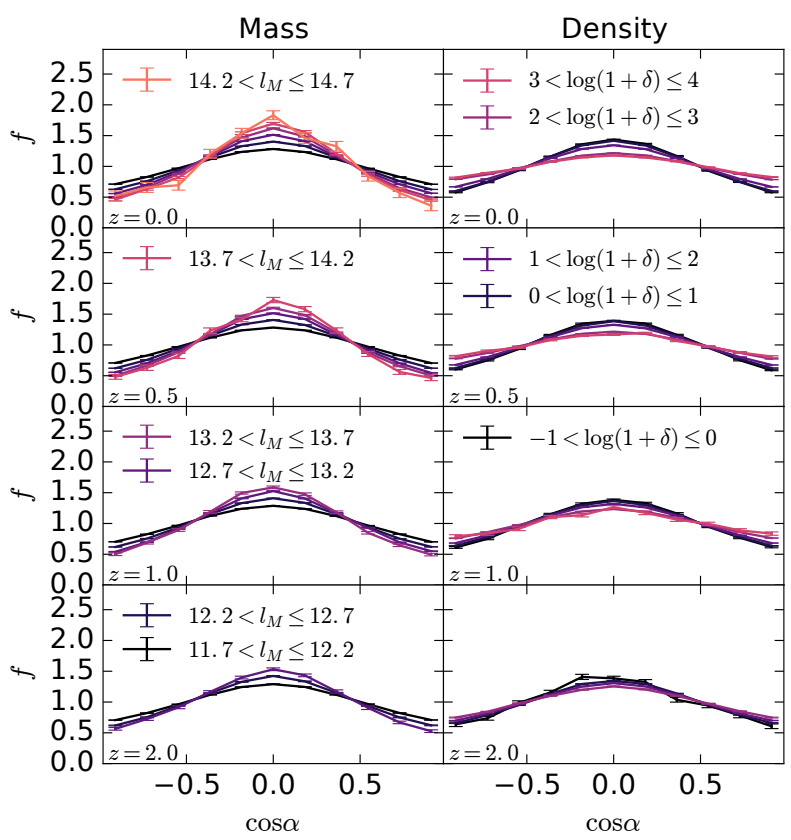

Figure 19. Effect of mass and density on the alignment of the direction of the neighbour and the spin of the target, as shown by the distribution of $\cos \alpha$, where $\alpha=\left(\boldsymbol{J}_{\mathrm{T}}, \boldsymbol{r}\right)$. Same legend as Fig. 17

of the position angle of the neighbour with the spin of the target $\left(\alpha=\left(\boldsymbol{J}_{\mathrm{T}}, \boldsymbol{r}\right)\right)$. As expected from Figs. 6 and 7 , the neighbour is preferentially located in the plane orthogonal to the spin of the target. The anti-alignment signal monotonically increases with the target mass, and decreases with the density. This is consistent with the findings of Welker et al.

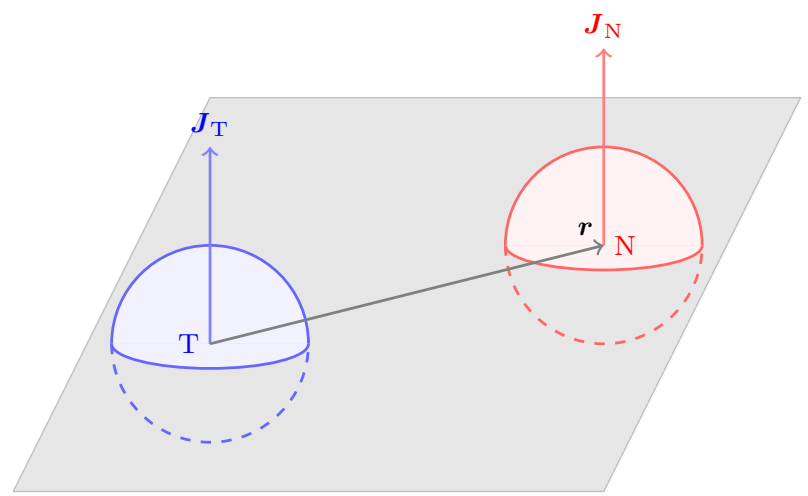

(a) Alignment of spins in the plane of interaction

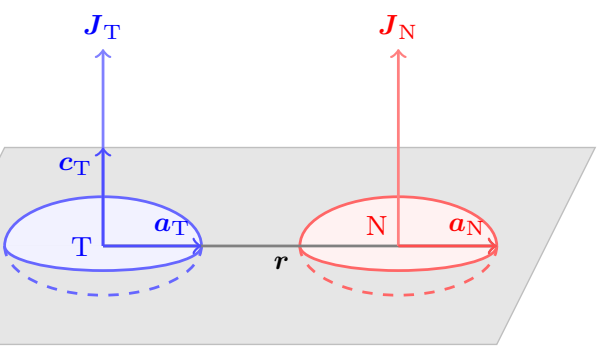

(b) Alignment of the major axes in the direction of the interaction

Figure 20. Summary: alignments of the spins and of the major axes

(2015), where the authors study the evolution of the angle $\theta_{1}=\left(\boldsymbol{c}_{\mathrm{T}}, \boldsymbol{r}\right)$ between the direction of the satellite and the minor axis of the central galaxy in the Horzizon-AGN hydrodynamical simulation. They found that as the satellite enters the virial radius, its direction becomes anti-aligned with the minor axis of the central. This is interesting as it is directly observable.

The dependence on mass and large-scale density is now more clear, as it was previously limited by the rather small dynamical range resulting from the choice of a constant number density of target. Using the whole range of masses available from the simulation enables us to better study the effects of mass, and the apparently weak mass dependency in the previous section appears to be caused by the small range of masses probed. However, in the previous section, the use of constant number density enabled us to study the redshift evolution of the alignment by following haloes that are statistically the same. These two approaches are therefore complementary.

\section{SUMMARY AND DISCUSSION}

We used the Horizon Run 4 cosmological $N$-body simulation to study the alignments of interacting haloes as a function of the mass and large-scale density, pair separation, as well as their time evolution. The very large volume of the simulation, combined with its large number of particles, enabled us to study the environmental effects on interactions. Our main findings are as follow.

- Interacting targets have a significantly lower spin and higher oblateness and sphericity parameters than all targets. The spin of interacting haloes decreases with increas- 
ing redshift up to $z \simeq 2$, with a faster decrease at lower densities (Fig. 1). Meanwhile, their prolateness and sphericity decrease with decreasing redshift and separation. The spin, sphericity, and oblateness increase with density, and are essentially constant with mass (Fig. 3).

- Interactions preferentially occur in the plane of rotation of the target haloes (the spins of targets are orthogonal to the directions of the neighbours, figs. 6 and 8 , and in the direction of the major axis of oblate haloes (Fig. 6). However, neighbours located in the direction of the spin of the target have their spin orthogonal (Fig. 13).

- The alignments of the position and main axis of the neighbour with the main axis of the target (Figs. 6 and 7) are stronger than those of the spins (Figs. 12. This can be understood by the fact that mergers can flip the spins, while the shape are more robust.

- We confirmed previous findings that the major axes of pairs are also aligned, and the spins of targets are aligned with the minor axes (Yang et al. 2006; Aragón-Calvo et al. 2007).

- Interacting pairs initially have anti-parallel spins, but the spins become parallel as time proceeds. The alignment signal is stronger at lower densities.

- The orbits are preferentially radial, initially with more approaching orbits. The signal become weaker as orbits become randomized.

- Pairs with non-radial trajectories initially have a random alignment between the spin of the neighbour and its orbital momentum, and the angles becomes more and more aligned as time goes on (Fig. 16).

- The signal of the alignment of the neighbour with major axis is stronger for more massive haloes, but only weakly depends on the large-scale density (Fig. 17). As for the spinspin alignment (Fig. 18), the signal is stronger for intermediate densities $(1<1+\delta<10)$, since at higher densities, haloes experience many interactions which can flip the spins. The anti-alignment of the position angle of the neighbour and the spin of the target (Fig. 19) can be tested observationally.

We now have a clear picture of how haloes interact, as summarised in Fig. 20. Starting with preferentially antiparallel or parallel spin orientations, on a radial orbit, haloes tend to have their neighbours aligned with their major axis, and orthogonal to their spin. The alignments become stronger and stronger in time, while the spin anti-parallel configuration disappears.

The alignments between the major axes and the pair separation $(\gamma$ and $\varepsilon)$ in Figs. 6 and 7 are in good qualitative agreement with observational work. For instance, Wang et al. (2009) studied the alignment of $\theta_{2}$, the angle between the projected major axis of the host group and the line connecting the host and neighbour groups, which corresponds to our definition of $\gamma$. Note however that in their case, the separation to the neighbour group may be larger than one virial radius away. They found a strong alignment signal, with a preference for $\theta \simeq 0$, corresponding to $\cos \gamma \simeq 1$ in our case. Moreover, when they restrict the sample to close pairs $\left(<3 R_{\mathrm{vir}, \mathrm{N}}\right)$, the alignment signal is stronger, which is also seen in our theoretical work.

Interestingly, Wang et al. (2009) did not detect any alignment signal for $\theta_{4}$, which corresponds to $\varepsilon$ in this work, even at smaller separations. Our work are in contradiction with these results, since we do find an alignment signal, albeit slightly weaker, for $\varepsilon$ as shown in Figs. 7 and 9 .

Moreover, the spin-spin and spin-position alignments in Fig. 12 is in good agreement with previous theoretical studies, such as Codis et al. (2012), who found that lowmass haloes have a spin parallel to their filaments, while massive ones are orthogonal. Even though we did not identify the filaments in this study, the direction of the nearest neighbour may be used as a proxy to that of the filament (Tempel \& Tamm 2015). We see that the anti-alignment between spin and neighbour direction becomes stronger as time passes (see Fig. 7 and 6. However, the masses of each bin are not fixed in time, but also grow. The strong antialignment at low-redshift, corresponding to higher masses, can be interpreted as massive haloes with a spin orthogonal to the filament, while the relatively weaker alignment at high redshift, corresponding to lower halo masses, may be due to the cases where low-mass haloes have their spin in the direction of the filament, pointing toward the neighbour. This qualitatively agrees with the mass-dependence of the spin-filament alignment found in Codis et al. (2012).

In a next step, we plan to study in more details the alignment of halo pairs with the large-scale structure and the cosmic web by using the Hessian of the smooth density field to characterise the environment (Hahn et al. 2007; ForeroRomero et al. 2009; Trowland et al. 2013).

However, this study was performed using a dark-matter only simulation. The observed shapes of galaxies come from the baryonic (stars) component, which needs to be modelled. Hahn et al. (2010) used cosmological hydrodynamic simulations to study the alignment of the galaxy and halo spins, and only found a weak alignment. In addition, it is interesting to see the effects of interaction on the morphology of galaxies, for instance to understand the effects of the hot gas halo on morphology transformation during interactions (Kim et al. 2013; Hwang et al. 2013; Hwang \& Park 2015). It is thus crucial to include hydrodynamics in our simulations to extend our analysis in comparison with observations. Direct comparison to previous observational work is difficult, because different definition of interaction are adopted. We plan to use existing observational data for comparison with our predictions using definition of galaxy interactions compatible with ours.

Moreover, the existence of an alignment at redshifts as high as $z=4$ suggests an origin in the initial density field. We will investigate this issue in a separate paper.

\section{APPENDIX A: EFFECT OF THE WEIGHTING IN THE INERTIA MATRIX}

The PSB algorithm truncates subhaloes at the tidal radius, which can affect the shape measurement. In the case of an isothermal density profile, the number of particles drops like $1 / r^{2}$. The number of particles in spherical shells with equal bin size is constant. Consequently, our method adopts an equal weighting in every shell. When applying a different weighing scheme, such as $1 / r^{2}$, to eq. (5), innermost particles are favoured. Of course our method is susceptible to outer boundary noise (numerical or varying with the subhalo definition). But in this study we want to study the interac- 


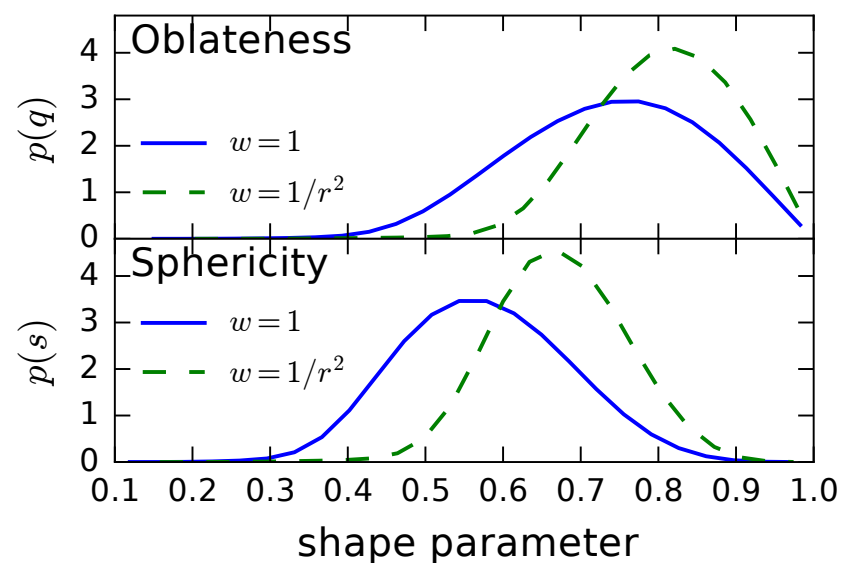

(a) Distribution of the oblateness (top) and sphericity (top) of target haloes for our two assignment schemes in the inertia tensor: $w=1$ (solid blue line) and $w=1 / r^{2}$ (green dashed line).

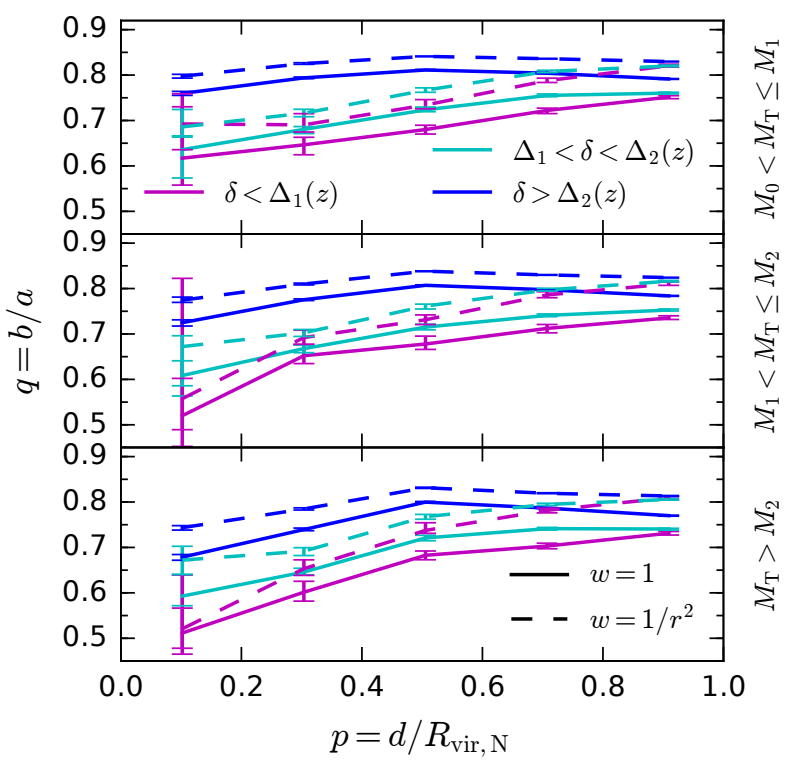

(b) Effect of the weighting scheme of the inertia tensor on the oblateness $q$ as a function of the normalized pair separation $p$ at $z=0$.

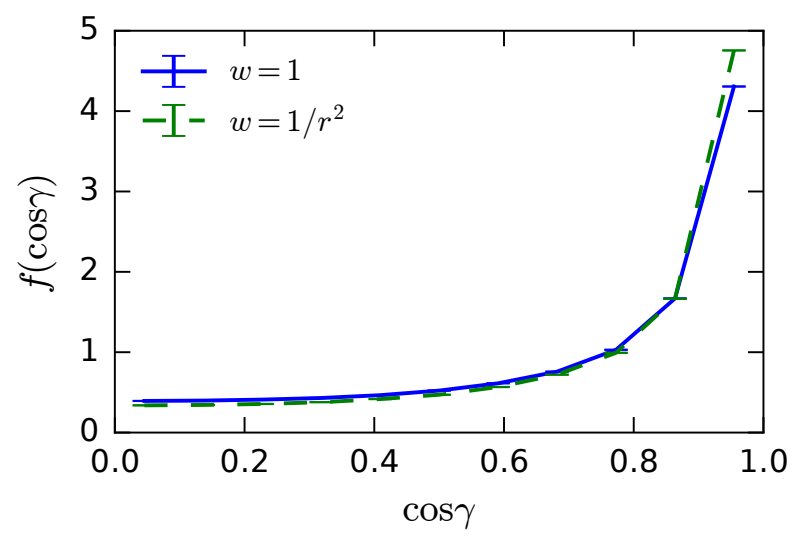

(c) Effect of the choice of the weighting of the inertia tensor on the alignment of the major axis of the target and the direction of the neighbour at $z=0$.

Figure A1. Effect of the weighting scheme of the inertia tensor on the distribution of the shape parameters (a) and on the alignment of the major axis with the direction of the neighbour (b). tions between the nearest neighbours, which may distort the halos shape in the outer boundary more seriously. Therefore, we adopt the shape tensor as defined in eq. (5). In this section, we address this issue by changing the weighting scheme in the calculation of the inertia matrix used to calculate the principal axes of the haloes. A more comprehensive study can be found in Bett (2012).

We consider the generalised inertia tensor

$$
\tilde{\mathbf{i}}_{i j}=\sum_{\alpha} w_{\alpha} \boldsymbol{x}_{\alpha, i} \boldsymbol{x}_{\alpha, j},
$$

where $\alpha$ is summed over all particles in the halo, and $i$ and $j$ are the considered directions. Our choice corresponds to $w_{\alpha}=1$. We compare it to another common choice, $w_{\alpha}=$ $1 / r_{\alpha}^{2}$. In the following we will drop the $\alpha$ for clarity.

Fig. A1(a) shows the effect of weighting in the inertia tensor on the distribution of the shape parameters $q$ and $s$. The $w=1 / r^{2}$ scheme gives more weight to the innermost particles, so its measurement is less sensitive to the external part of the halo. The $w=1 / r^{2}$ yields a larger mean sphericity and smaller dispersion in both cases. This shows that, even though PSB truncates the subhaloes at the tidal radius, the sphericity is overall dominated by the innermost particles, therefore our results are weakly affected by the effects of the subhalo finder and the truncation to the tidal radius. Panel (b) shows the effect of the weighting scheme on the evolution of the oblateness parameter with the reduced pair separation $p$. The colors are the same as in Fig. 4b, but the solid line shows the $w=1$ case and the dashed line the $w=1 / r^{2}$ weighting scheme. While the actual oblateness is higher for the $w=1 / r^{2}$ case, as expected from the distribution in panel (a), the trend is similar in all cases. Fig. A1(c) shows the effect of weighting the inertia matrix on the alignment of the major axis of the target and the direction of the neighbour, defined as angle $\gamma$, at $z=0$, and for all interacting targets. Our choice of $w=1$ is shown in blue, solid line, while $w=1 / r^{2}$ is shown in green, dashed line. The alignment signal is slightly stronger when $w=1 / r^{2}$ is considered.

Our choice of $w=1$ is thus a conservative definition for the inertia matrix regarding the alignment.

\section{Acknowledgements}

We thank KIAS Center for Advanced Computation for providing computing resources.

\section{REFERENCES}

Ahn J., Kim J., Shin J., Kim S. S., Choi Y.-Y., 2014, Journal of Korean Astronomical Society, 47, 77

Aragon-Calvo M. A., Yang L. F., 2014, MNRAS, 440, L46

Aragón-Calvo M. A., van de Weygaert R., Jones B. J. T., van der Hulst J. M., 2007, ApJ, 655, L5

Aryal B., Paudel S., Saurer W., 2007, MNRAS, 379, 1011

Bett P., 2012, MNRAS, 420, 3303

Bett P., Eke V., Frenk C. S., Jenkins A., Helly J., Navarro J., 2007, MNRAS, 376, 215

Bond J. R., Kofman L., Pogosyan D., 1996, Nature, 380, 603

Boylan-Kolchin M., Springel V., White S. D. M., Jenkins A., Lemson G., 2009, MNRAS, 398, 1150

Brainerd T. G., 2005, ApJ, 628, L101 
Cervantes-Sodi B., Hernandez X., Hwang H. S., Park C., Le Borgne D., 2012, MNRAS, 426, 1606

Chen Y.-C., et al., 2015, MNRAS, 454, 3341

Chisari N., et al., 2015, MNRAS, 454, 2736

Codis S., Pichon C., Devriendt J., Slyz A., Pogosyan D., Dubois Y., Sousbie T., 2012, MNRAS, 427, 3320

Doroshkevich A. G., 1970, Astrofizika, 6, 581

Dubois Y., et al., 2014, MNRAS, 444, 1453

Forero-Romero J. E., Hoffman Y., Gottlöber S., Klypin A., Yepes G., 2009, MNRAS, 396, 1815

Forero-Romero J. E., Contreras S., Padilla N., 2014, MNRAS, 443, 1090

Hahn O., Carollo C. M., Porciani C., Dekel A., 2007, MNRAS, 381,41

Hahn O., Teyssier R., Carollo C. M., 2010, MNRAS, 405, 274

Hoffman Y., Metuki O., Yepes G., Gottlöber S., Forero-Romero J. E., Libeskind N. I., Knebe A., 2012, MNRAS, 425, 2049

Hoyle F., 1951, in Problems of Cosmical Aerodynamics. p. 195

Hwang J.-S., Park C., 2015, ApJ, 805, 131

Hwang J.-S., Park C., Choi J.-H., 2013, Journal of Korean Astron. Soc., 46, 1

Joachimi B., et al., 2015, Space Sci. Rev.,

Kim J., Park C., 2006, ApJ, 639, 600

Kim E., Choi Y.-Y., Kim S. S., 2013, Journal of Korean Astronomical Society, 46, 33

Kim J., Park C., L'Huillier B., Hong S. E., 2015, Journal of Korean Astronomical Society, 48, 213

L'Huillier B., Park C., Kim J., 2014, New A, 30, 79

L'Huillier B., Park C., Kim J., 2015, MNRAS, 451, 527

Laigle C., et al., 2015, MNRAS, 446, 2744

Lee J., 2011, ApJ, 732, 99

Lee J., Erdogdu P., 2007, ApJ, 671, 1248

Lee J., Pen U.-L., 2000, ApJ, 532, L5

Lee J., Pen U.-L., 2002, ApJ, 567, L111

Lee J., Kang X., Jing Y. P., 2005, ApJ, 629, L5

Libeskind N. I., Hoffman Y., Forero-Romero J., Gottlöber S., Knebe A., Steinmetz M., Klypin A., 2013a, MNRAS, 428, 2489

Libeskind N. I., Hoffman Y., Steinmetz M., Gottlöber S., Knebe A., Hess S., 2013b, ApJ, 766, L15

Monaghan J. J., Lattanzio J. C., 1985, A\&A, 149, 135

Park C., Choi Y.-Y., 2009, ApJ, 691, 1828

Park C., Choi Y.-Y., Vogeley M. S., Gott III J. R., Blanton M. R., SDSS Collaboration 2007, ApJ, 658, 898

Park C., Gott III J. R., Choi Y.-Y., 2008, ApJ, 674, 784

Peebles P. J. E., 1969, ApJ, 155, 393

Porciani C., Dekel A., Hoffman Y., 2002, MNRAS, 332, 325

Schneider M. D., Frenk C. S., Cole S., 2012, J. Cosmology Astropart. Phys., 5, 30

Shao S., Cautun M., Frenk C. S., Gao L., Crain R. A., Schaller M., Schaye J., Theuns T., 2016, MNRAS, 460, 3772

Springel V., White S. D. M., Tormen G., Kauffmann G., 2001, MNRAS, 328, 726

Springel V., et al., 2005, Nature, 435, 629

Tempel E., Tamm A., 2015, A\&A, 576, L5

Trowland H. E., Lewis G. F., Bland-Hawthorn J., 2013, ApJ, 762, 72

Velliscig M., et al., 2015a, MNRAS, 453, 721

Velliscig M., et al., 2015b, MNRAS, 454, 3328

Wang Y., Yang X., Mo H. J., Li C., van den Bosch F. C., Fan Z., Chen X., 2008, MNRAS, 385, 1511

Wang Y., Park C., Yang X., Choi Y.-Y., Chen X., 2009, ApJ, 703, 951

Welker C., Dubois Y., Pichon C., Devriendt J., Chisari E. N., 2015, preprint, (arXiv:1512.00400)

White S. D. M., 1984, ApJ, 286, 38

Yang X., van den Bosch F. C., Mo H. J., Mao S., Kang X., Weinmann S. M., Guo Y., Jing Y. P., 2006, MNRAS, 369, 1293
Zhang Y., Yang X., Faltenbacher A., Springel V., Lin W., Wang H., 2009, ApJ, 706, 747

Zhang Y., Yang X., Wang H., Wang L., Mo H. J., van den Bosch F. C., 2013, ApJ, 779, 160

Zhang Y., Yang X., Wang H., Wang L., Luo W., Mo H. J., van den Bosch F. C., 2015, ApJ, 798, 17

de Lapparent V., Geller M. J., Huchra J. P., 1986, ApJ, 302, L1 